\title{
Transits against Fainter Stars: The Power of Image Deconvolution
}

\author{
Penny D. Sackett ${ }^{1}$, Michaël Gillon ${ }^{2}$, \\ Daniel D. R. Bayliss ${ }^{1}$, David T. F. Weldrake ${ }^{3}$ and Brandon Tingley ${ }^{4}$ \\ ${ }^{1}$ Research School of Astronomy and Astrophysics, Australian National University, \\ Mount Stromlo, Australia \\ email: Penny.Sackett@anu.edu .au \\ ${ }^{2}$ Observatoire de l'Université de Genève, Switzerland \\ ${ }^{3}$ Harvard-Smithsonian Center for Astrophysics, USA \\ ${ }^{4}$ Instituto de Astrofisica de Canarias, Spain
}

\begin{abstract}
Compared to bright star searches, surveys for transiting planets against fainter $(V=$ 12-18) stars have the advantage of much higher sky densities of dwarf star primaries, which afford easier detection of small transiting bodies. Furthermore, deep searches are capable of probing a wider range of stellar environments. On the other hand, for a given spatial resolution and transit depth, deep searches are more prone to confusion from blended eclipsing binaries. We present a powerful mitigation strategy for the blending problem that includes the use of image deconvolution and high-resolution imaging. The techniques are illustrated with Lupus-TR-3 and very recent IR imaging with PANIC on Magellan. The results are likely to have implications for the CoRoT and KEPLER missions designed to detect transiting planets of terrestrial size.
\end{abstract}

\section{Introduction}

Most searches for transiting extrasolar planets fall into two broad categories: very wide-field searches targeting bright $(V<12)$ stars, and narrower field, pointed, densefield observations monitoring fainter $(V>14)$ stars (Fig. 1). Surveys of brighter stars have the advantage of more efficient spectroscopic follow-up due to the larger fluxes of their candidates. Fainter searches are typically more efficient in the discovery phase, using less telescope time to densely monitor a similar number of dwarf star targets.

The space-based CoRoT (Barge et al., this volume) and KEPLER (Borucki et al., this volume) missions bridge part of this gap, pointing at particular dense stellar fields at low Galactic latitude, with the expectation that most of their prime target stars will have $12<V<14$. This middle range is their "sweet spot" because the stellar mass function ensures that most dwarf hosts will lie at the faint end, while the best photometry required to search for small planets is achieved at the bright end. Certainly the transiting planets reported by the CoRoT team to date, all with Jovian sizes, have host stars in this magnitude range (Barge et al. 2008b; Alonso et al. 2008; Aigrain et al. 2008).

Stars of this magnitude and fainter are increasingly more likely to be blended with foreground or background stars of similar brightness. Furthermore, the ability to perform spectroscopic tests to rule out the possibility that a blended eclipsing binary is masquerading as a transiting planet becomes increasingly difficult as the host star becomes fainter. Since Jovian-sized planets generate a $\sim 1 \%$ dip in host brightness when transiting a Sun-like star, a (totally) eclipsing stellar binary (EcB) system can be nearly five magnitudes fainter than a random blended neighbour along the line-of-sight and still generate a Jovian-like transit signal against the bright blended composite. For the same 


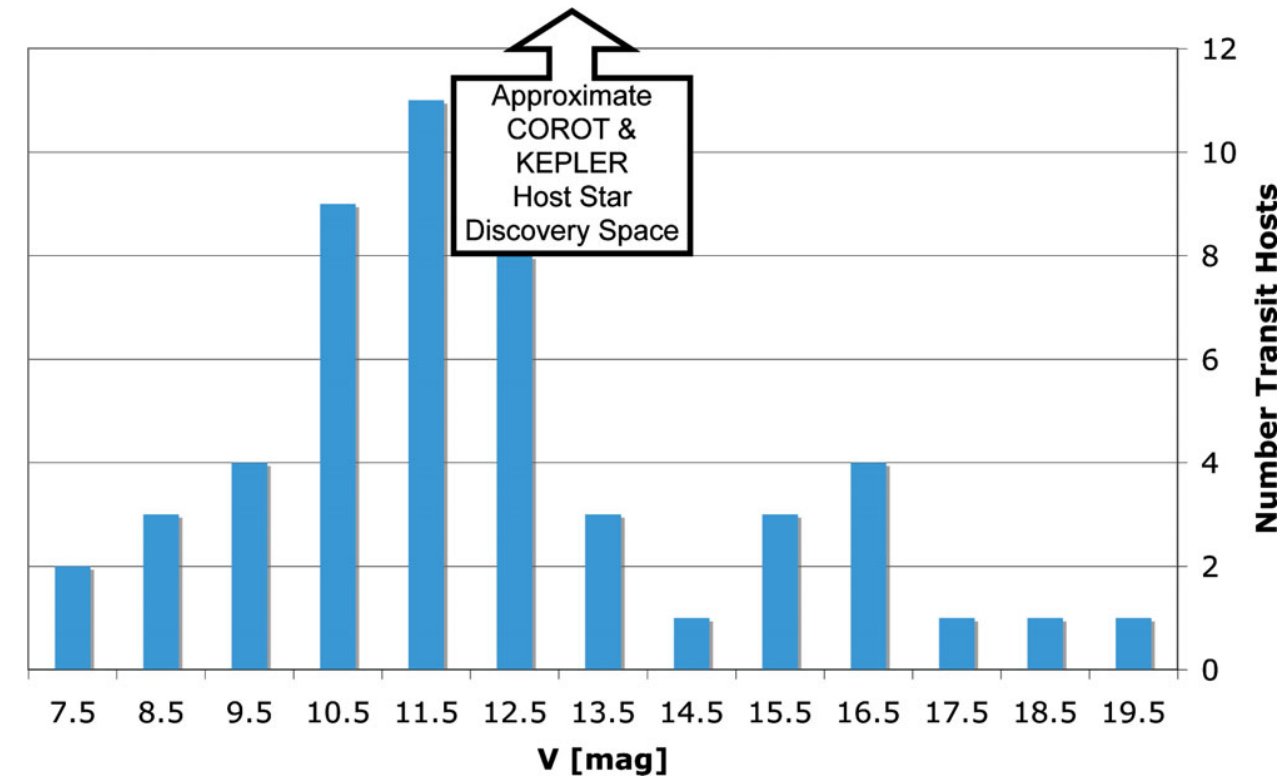

Figure 1. A histogram of the $V$ magnitude of host stars reported to have transiting planets.

reason, surveys for terrestrial-sized planets must be able to exclude possible blended EcB up to ten magnitudes fainter than their survey targets. Clearly, this is an issue that the CoRoT and KEPLER teams will have to face in confirming planets whose apparent masses are too small to yield a robust radial velocity signature.

\section{The Lupus Survey and Lupus-TR-3b}

We were led to consider the issue of possible confusion with an EcB in follow-up work directed at the prime planetary transit candidate Lupus-TR-3b (Weldrake et al. 2008), found in our initial deep survey of the Lupus region. Like the CoRoT (Serpens Cauda: $l=30, b \sim 0 ;$ Monoceros: $l=215, b \sim 0$ ) and KEPLER (Cygnus: $l=76, b=13$ ) fields, our Lupus survey was performed in a dense Galactic field (Lupus: $l=331, b=11$ ).

In our survey, we monitored about 110,000 stars over a 0.66 -square-degree field in Lupus for 53 nights in June of 2005 and 2006 with the ANU 40-Inch Telescope equipped with a wide-field imager at Siding Spring Observatory (SSO) in Australia (Weldrake et al. 2007). The resulting 1783 exposures produced photometry to a precision of better than $0.025 \mathrm{mag}$ ( $\mathrm{rms}$ ) for $\sim 16,000$ stars. The photometry was produced using an image subtraction technique, followed by SYSREM (Tamuz et al. 2005) to remove systematics (red noise) common to a large number of stars in the field. The BLS detection scheme of Kovács et al. (2002) was then used to identify promising candidates for the host stars of transiting planets. This initial two-year survey is being extended in time to yield the SuperLupus Survey (see Bayliss et al. in this volume), and will increase the sensitivity to longer-period transiting planets.

The initial photometric selection process produced six candidates in the Lupus field, whose basic characteristics are listed in Table 1. More details will be forthcoming in a subsequent publication (Bayliss et al., in prep.). All candidates were detected at a high level of significance, with a large number of in-transit photometric measurements, and 
Table 1. Characteristics of Lupus Survey Candidates

\begin{tabular}{lcccc}
\hline Host Star & V $[\mathbf{m a g}]$ & Detection S/N & \# In-transit Points & $\eta$ Diagnostic $^{2}$ \\
\hline Lupus-TR-1 & 14.6 & 20.9 & 70 & 1.3 \\
Lupus-TR-2 & 14.8 & 26.3 & 59 & 1.0 \\
Lupus-TR-3 & $\mathbf{1 7 . 4}$ & $\mathbf{2 4 . 2}$ & $\mathbf{1 2 5}$ & $\mathbf{0 . 7}$ \\
Lupus-TR-4 & 16.3 & 23.2 & 141 & 1.2 \\
Lupus-TR-5 & 17.4 & 13.6 & 66 & 0.8 \\
Lupus-TR-6 & 17.4 & 15.0 & 57 & 0.6 \\
\hline
\end{tabular}

Notes:

${ }^{1}$ The detection $\mathrm{S} / \mathrm{N}$ is a measure of the significance of the size of collective transit signal.

${ }^{2}$ The $\eta$ diagnostic is unity or below for likely planetary transits (see Tingley \& Sackett 2005 ).

depth-duration-period properties, as measured by the $\eta$ diagnostic (Tingley \& Sackett 2005), that made them plausible transiting planet signatures.

After further scrutiny and follow-up observations, however, only Lupus-TR-3 remained as a plausible candidate. This K1 dwarf exhibits a $1.3 \%$ dip of about 2.6 hour duration every $P=3.91405$ days. Subsequent radial velocity measurements taken with the MIKE echelle spectrograph on Magellan I displayed a confirming radial velocity signature of $K=114 \pm 25 \mathrm{~m} / \mathrm{s}$, appropriately in phase with the transit. The planet, Lupus-TR-3b, thus has derived parameters of $M_{p}=0.81 \pm 0.18 M_{J}, R_{p}=0.89 \pm 0.07 R_{J}$, yielding a Jovian-like density of $\rho_{p}=1.4 \pm 0.4 \mathrm{gm} / \mathrm{cm}^{3}$ (Weldrake et al. 2008).

This might have been the end of the story, had we not simultaneously been pursuing image deconvolution as a method to discover possible close neighbours in the vicinity of promising candidates.

\section{A (De)Convoluted Cautionary Tale}

The long train of monitoring images used to discover Lupus-TR-3b were obtained at $\mathrm{SSO}$, a site with only moderate atmospheric seeing conditions. Furthermore, the ANU 40Inch Telescope is known to have guider issues, and so the 5-minute frames were taken unguided. As a result, a typical image would have a stellar point spread function (PSF) with a full width at half maximum of $\mathrm{FWHM} \approx 2^{\prime \prime}$. Although this is considerably better image quality than obtained by wide-field transit surveys against bright stars, we decided to apply image deconvolution to the immediate Lupus-TR-3 field as an experiment aimed at the possibility of finding a line-of-sight "neighbour" within the typical FWHM.

For this purpose, we used a package originally developed for use in extragalactic lensing, but later optimised for point sources. This algorithm, DECPHOT (Gillon et al. 2006; Magain et al. 2007), weights the PSF properly in the statistical sense, and uses all of the information on the image to achieve the best deconvolution. On the other hand, it is CPU intensive, so that it is generally applied only to "postage stamp" areas around the immediate object of interest rather than to a complete CCD mosaic.

An initial DECPHOT application to our SSO images revealed two possible neighbours, dubbed stars $B$ and $C$ (Fig. 2). Star $C$, located 2.2" from Lupus-TR-3, is 5.6 V magnitudes fainter than the target, and thus could not be responsible for the transit signature even if it was an EcB that fully eclipsed its primary. Star $B, 1.7^{\prime \prime}$ from the target is 4.1 $V$ magnitudes fainter, and could thus, in principle, be a planet-pretender.

However, by performing photometry on a time-sequence of DECPHOT deconvolved SSO images covering both in- and out-of-transit epochs, we were able to demonstrate that Lupus-TR-3 was undergoing a partial eclipse, not Star B (Weldrake et al. 2008). 

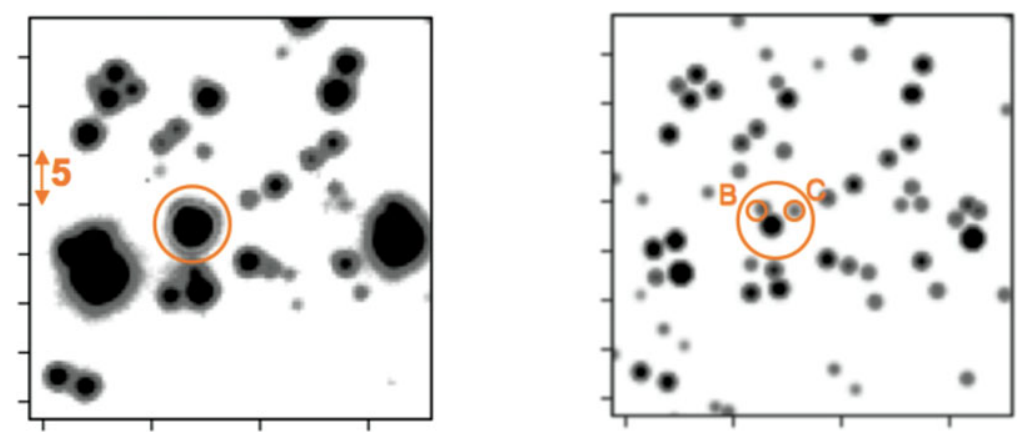

Figure 2. Left: A typical image from the ANU 40-inch, for which Lupus-TR-3 is the circled object. Right: The same SSO field, to the same scale, shown after application of DECPHOT. Two neighbours, stars $B$ and $C$, were indicated.

Again, the story might have ended, had we not obtained a good seeing $\left(\mathrm{FWHM} \sim 0.7^{\prime \prime}\right)$ image of the Lupus-TR-3 field in the $Y$ band ( $\sim 1.1$ micron) with the PANIC infrared imager on Magellan II, kindly provided by the Las Campanas Observatory staff. Our aim was to check the positions and relative brightness of neighbours predicted by the deconvolution of SSO images.

The Magellan $Y$ band image (Fig. 3) indeed indicated that DECPHOT had correctly identified blended neighbours $B$ and $C$, but also indicated a third star, $D, 1.8^{\prime \prime}$ from the target that was $4.3 Y$ magnitudes fainter than the Lupus-TR-3, and fainter still in $V$ - too faint to be a culprit. Naturally curious, however, we then applied DECPHOT to the high-quality PANIC image. To our surprise, the deconvolution predicted yet another

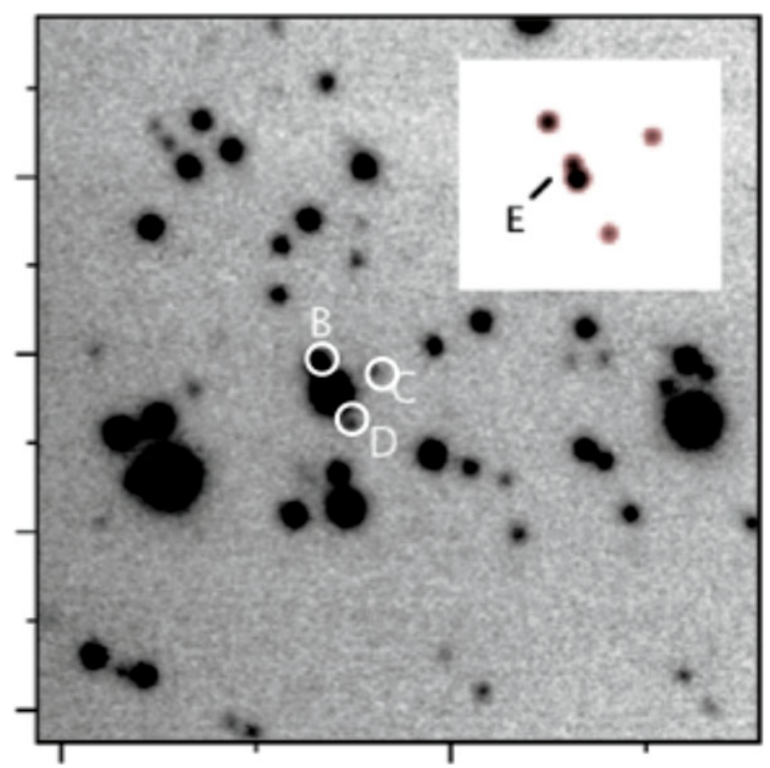

Figure 3. Main: A Magellan $Y$-band PANIC image of the Lupus-TR-3 field reveals stars $B$ and $C$, both predicted by DECPHOT analysis of SSO images, and also a very faint neighbour $D$. Insert: Subsequent deconvolution of PANIC frames then predicted a very near neighbour, labelled $E$, a possible, but unlikely, planet pretender. 


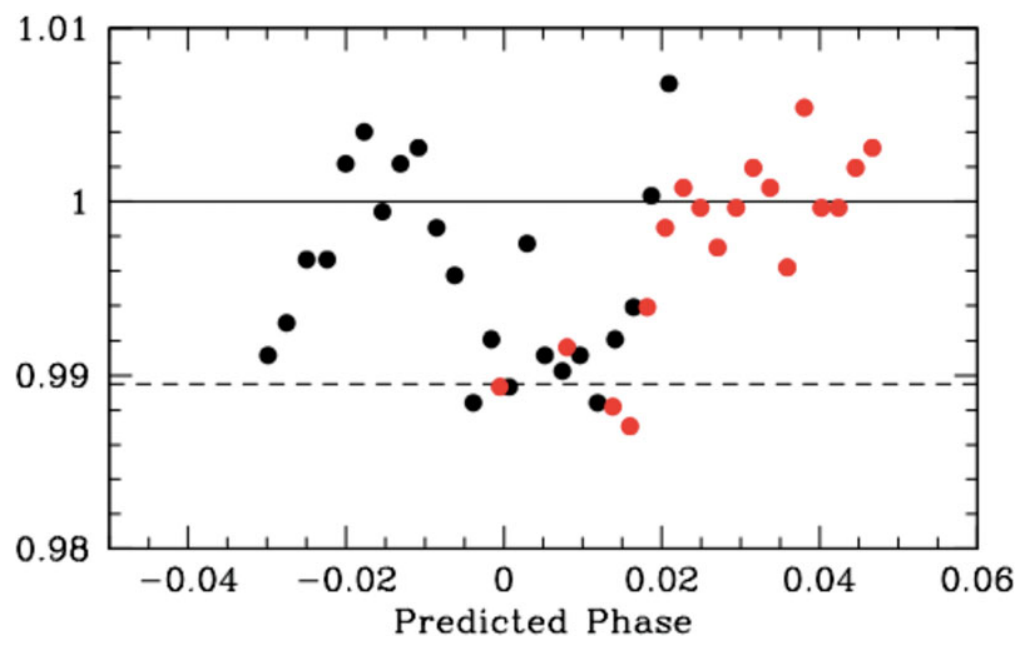

Figure 4. Binned, "on-mountain" aperture photometry on PANIC near-IR images of Lupus-TR-3. The first two points were affected by "poor" Las Campanas seeing of 1 ". Black (darker) points were taken in April 2008, and red (lighter) points were obtained two periods later in May.

neighbour, this one, star $E, 2.8$ magnitudes fainter in $Y$ than the target, and only $0.4^{\prime \prime}$ distant. The inability of DECPHOT to identify it in our broad $V+R$ images from Siding Spring seemed to indicate that star $E$ must have $V>21$, and thus would have to suffer an eclipse of $36 \%$ or more to create a spurious transit signal. Its faintness, coupled with the unusually parameters required for an EcB to mimic our signal (see Weldrake et al. 2008), make star $E$ an unlikely, if possible, false positive.

Nevertheless, in order to check this possibility, and to provide yet another test of DECPHOT predictions, we applied for PANIC imaging time on Magellan over two epochs that would cover both in- and out-of-transit points. The aim was to perform photometry on a time-series of deconvolved PANIC images capable of separating the original target star from the putative neighbour $E$ in order to directly discern, as we had earlier for star $B$, which was undergoing a partial eclipse.

These data were obtained in April and May of 2008, producing over 200 frames in $Y$ band with a typical PSF of $0.55^{\prime \prime}$. A very rough reduction on the mountain (see Fig. 4) indicates that we have captured the transit to within the uncertainties on the transit timing predicted a year earlier.

After a first data reduction, small $(250 \times 250$ pixel $)$ stamps around Lupus-TR-3 were processed using DECPHOT, using only the best of the April 2008 data, namely those with seeing below $0.5^{\prime \prime}$. (Note that the images are still well-sampled, as PANIC has pixels that translate into $0.125^{\prime \prime}$ on the sky.) These data represent about $50 \%$ improvement in image quality compared to our earlier PANIC snapshot of the field.

The results are surprising. While neighbours $B, C$ and $D$ are all seen at their DECPHOT predicted positions and brightness, it appears that (non-ecliping) star B is actually two stars, here labelled $B 1$ and $B 2$ (see Fig. 5). While it is unclear whether the faint, close neighbour $E$ originally predicted from DECPHOT analysis of our earlier, somewhat poorer PANIC frame, is actually present, it does seem clear that the primary target, Lupus-TR-3 itself has two components, $A 1$ and $A 2$ ! The component $A 2$ is only 2.3 times fainter than $A 1$ according to our preliminary analysis, and the two are separated by only $0.25^{\prime \prime}$ on the sky, making it conceivable that they form a wide, physical binary. Our 


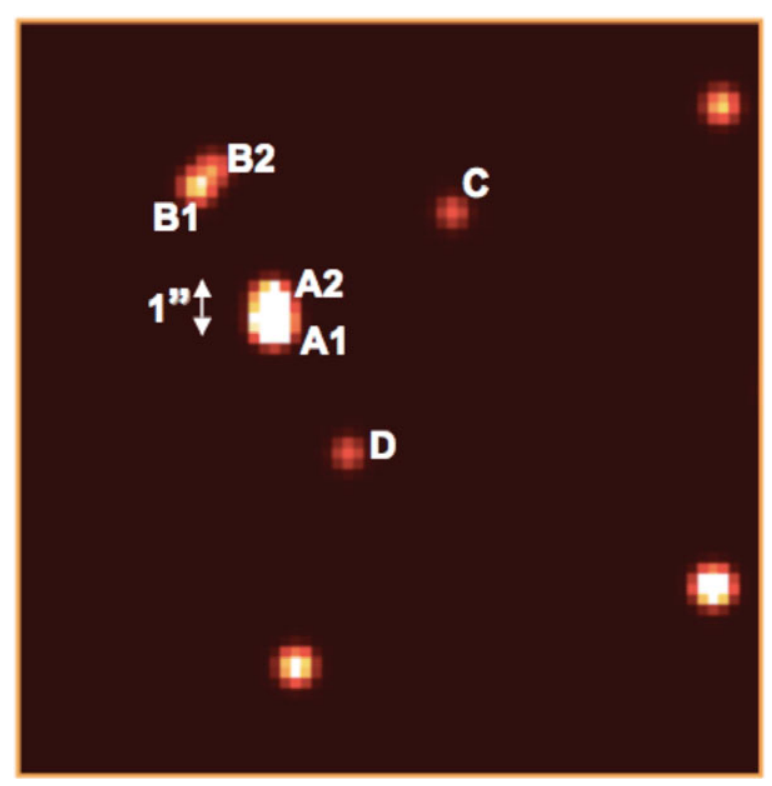

Figure 5. Preliminary DECPHOT analysis of a subset of the PANIC frames of the Lupus-TR-3 field reveal the expected faint stars $C$ and $D$, but also that the (non-eclipsing) star $B$ is actually composed of two components, as is the primary target. The length of the arrow denotes $1^{\prime \prime}$ on the sky, indicating that this image is considerably zoomed compared to those in Figs. 2 and 3.

original Lupus-TR-3 target, then, is composed of at least six, and perhaps seven, individual objects.

We are now in the process of undertaking a more detailed, thorough study of all the PANIC frames from Magellan of the Lupus-TR-3 field in order to understand whether (1) star $E$ is present, and (2) star $A 1, A 2$ or possibly $E$ is eclipsing. Either $A 1$ or $A 2$ could host a transiting planet, albeit one of different planetary characteristics than were derived under the assumption that they formed a single star (Sackett et al. 2008). In the meantime, we offer our experience as a cautionary tale for all those who might imagine that the target they are observing, especially in fields of low Galactic latitude, is a single star with no faint, possibly EcB, blended neighbours, capable of mimicking a planetary transit.

\section{Conclusions}

Our experience with deep, high-spatial resolution imaging of the field of the planetary transit host star Lupus-TR-3 suggest that:

- It is vital to constrain the presence of blended line-of-sight neighbours up to 5 magnitudes fainter than stars thought to host Jovian-sized transiting planets, and up to 10 magnitudes fainter than those thought to host terrestrial-sized transiting planets. Faint, eclipsing stellar binaries may otherwise cause an unacceptable level of false positives in searches for transiting extrasolar planets.

- Fields of low Galactic latitude ( $b \sim 10 \mathrm{deg}$ or less) have a relatively high probabilities of such impostors and confusing contaminants.

- Even faint, undetected, non-EcB neighbours will influence the inferred radius of detected planets, by causing an underestimation of the true transit depth, and thus an underestimation of the true radius. 
- The CoRoT and KEPLER missions may be particularly susceptible to this contamination in their search for terrestrial-sized planets.

- Image deconvolution coupled with high-spatial resolution imaging is a powerful tool for locating and studying many of these annoying neighbours.

\section{References}

Aigrain, S., et al. 2008, accepted to AESA, arXiv:0807.3767

Alonso, R., et al. 2008, A\&A, 482, L21

Barge P., et al. 2008b, A\&SA, 482, L17

Gillon, M., et al. 2006, A\&BA, 459, 249

Kovács, G., Zucker, S., \& Mazeh, T. 2002, A\&A, 391, 369

Magain, P., 2007, A\&SA, 461, 373

Tamuz, O., Mazeh, T., \& Zucker, S. 2005, MNRAS, 356, 1466

Tingley, B. \& Sackett, P.D. 2005, ApJ, 627, 1011

Weldrake, D. T. F., et al. 2007, Transiting Extrapolar Planets Workshop, 366, 90

Weldrake, D. T. F., et al. 2008, ApJ, 675, L37 